\title{
Radiographic Outliers, Revisions, and Functional Outcomes in Fixed-Bearing Medial Unicompartmental Arthroplasty
}

\author{
Colin Harris ${ }^{1}$, Evan R. Deckard, ${ }^{2}$ Mary Ziemba-Davis, ${ }^{3}$ \\ Kevin A. Sonn, ${ }^{2,3}$ R. Michael Meneghini ${ }^{2,3}$ \\ ${ }^{1}$ Indiana University School of Medicine; ${ }^{2}$ Indiana University School of Medicine, Department of \\ Orthopaedic Surgery; ${ }^{3}$ Indiana University Health Physicians, IU Health Hip \& Knee Center
}

\section{Background and Hypothesis:}

Unicompartmental Knee Arthroplasty (UKA) is a technically demanding procedure vulnerable to errors in component positioning. Recent studies suggest robotic-assistance improves precision of implant placement, minimizes outliers, and improves survivorship. However, we have previously demonstrated an experienced surgeon can match robotic accuracy. This study evaluated revision rates and functional outcomes of radiographic outliers in manual UKA.

\section{Experimental Design or Project Methods:}

A retrospective review of 222 consecutive fixed-bearing medial UKAs was conducted. Implant positioning and alignment were assessed radiographically measuring tibial coronal (TCA), femoral coronal (FCA), tibial sagittal (TSA) and femoral sagittal (FSA) angles as well as implant overhang. UCLA Activity Level and all-cause survivorship were evaluated. Student's t-test, Chi square, and Kaplan Meier curves were used in analyses with alpha $\leq .05$ designating statistical significance.

\section{Results:}

Using previously published alignment goals, our manual UKAs achieved the following targets: $92 \%$ for TCA, $100 \%$ for FCA, $88 \%$ for TSA, and $100 \%$ for FSA. For implant overhang, 100\% met medial, anterior, and posterior targets. Our manual UKAs achieved desired alignment and overhang goals more frequently than previously published manual success. Survivorship free from aseptic revision in this study was $96 \%$ at 8.5 years. Additionally, there was no difference in UCLA Activity Level improvement comparing outliers and non-outliers ( $p \geq 0.159)$.

\section{Conclusion and Potential Impact:}

Manual UKAs performed by an experienced surgeon showed improved success in achieving alignment and implant overhang goals compared to published manual UKA data and similar success compared to published robotic-assisted UKA data. We found no differences in revision rates or functional outcomes between outliers and non-outliers using previously published targets. For robotic assistance to be cost effective, it must demonstrate improved outcomes or survivorship when compared to manual UKAs. Our results contradict recently published claims that manual alignment outliers and failure rates fail to meet expectations for UKA. 